\title{
Taste cell is abundant in the expression of ACE2 receptor of 2019-nCoV
}

\author{
Qi Han ${ }^{1}$, Jiakuan Peng ${ }^{1}$, Hao $\mathrm{Xu}^{1 *}$, Qianming Chen ${ }^{1,2}$
}

\begin{abstract}
1. State Key Laboratory of Oral Diseases, National Clinical Research Center for Oral Diseases, Chinese Academy of Medical Sciences Research Unit of Oral Carcinogenesis and Management, West China Hospital of Stomatology, Sichuan University, Chengdu, Sichuan, 610041, P.R. China

2. Key Laboratory of Oral Biomedical Research of Zhejiang Province, Affiliated Stomatology Hospital, Zhejiang University School of Stomatology
\end{abstract}

*Correspondence: Hao Xu(xhstatis@hotmail.com)

\begin{abstract}
The infected and fatal cases of Coronavirus Disease 2019 (COVID-19) keep increasing around the world, to explore the infection routes and pathogenesis of 2019-nCoV could be meaningful for prevention and treatment of COVID-19. Previous studies showed that the oral cavity is at potentially high risk of 2019-nCoV infection. The ACE2 receptor of 2019-nCoV was reported could express on oral epithelium and salivary glands, and 2019-nCoV could be detected in patients' saliva. Recently, the amblygeustia were found to widely exist in the COVID-19 patients. To explore the potential mechanism of amblygeustia, we performed further analysis via independent in-house single-cell profiles. Our results showed that ACE2 was inclined to express in taste cells, which indicated that 2019-nCoV may invade into taste cells at the early stage of COVID-19, and lead to the amblygeustia of patients. Above findings about the 2019$\mathrm{nCoV}$ and COVID-19 in oral cavity are valuable and enlightening for future epidemic prevention strategy.
\end{abstract}




\section{Introduction}

The Corona Virus Disease 19 (COVID-19) has developed into a global pandemic, and the epidemic situation was still deteriorating so far. After recent months of hard work, a great progress was made in acknowledging the prevention, control and treatment of COVID-19. While, it still takes some time to develop specific medication and vaccine. The heterogeneity of symptoms has been one of the difficulties in prevention and control of COVID-19 at this stage. Part of COVID-19 patients didn't present typical symptoms such as fever and cough, even many patients remained asymptomatic after confirmed infection ${ }^{1}$. These patients may be the loopholes of prevention and control, increasing additional risk of continuous spreading. As the research of 2019-nCoV going deeply, the virus was found that could invade other organs besides lung, such as heart, kidney and intestines ${ }^{2}$. Recent reports found that oral cavity could be functional vector for virus infection, and $2019-\mathrm{nCoV}$ could be detected in saliva ${ }^{3,4}$. More interestingly, oral symptoms were reported in the COVID-19 patients, especially amblygeustia, Which may point a new direction and thought for 2019-nCoV prevention and control ${ }^{5}$. Therefore, in this paper, we briefly summarized recent 2019-nCoV related studies in oral cavity field, and performed a future analysis to explore the potential mechanism of amblygeustia based on our previous study.

\section{Receptor of 2019-nCoV could express on the oral cavity}

Our previous research has explored the expression of ACE2 receptor of 2019-nCoV on the oral mucosa with public databases and independent in-house single-cell transcriptomes ${ }^{3}$. This result demonstrated that the ACE2 could express on oral epithelium, especially high expression on the dorsum linguae. The finding indicated that the oral epithelium could be the potential infection route of the COVID-19. Then, another study reported that the ACE2 could express on the salivary glands, which confirmed the oral cavity is a potentially high risk for $2019-\mathrm{nCoV}$ infectious susceptibility ${ }^{5}$.

\section{9-nCoV could be detected in saliva}

Recently, further studies claimed the 2019-nCoV could be detected in COVID-19 patients' saliva and indicated the potential risk of virus infection via saliva ${ }^{4-6}$. According to the results of Chen et al., the detectable rate of $2019-\mathrm{nCoV}$ in patients' saliva was high, especially in the critical patients, which suggested that the detectable rate in saliva could be an indicator to reflect 
the illness state of patients ${ }^{5}$. Meanwhile, a cohort study of To et al. showed the feasibility of using saliva samples for viral load monitoring ${ }^{4}$. As the acquisition of saliva is quite convenient and noninvasive, those findings showed the potential value of saliva testing to detect and evaluate the illness state of COVID-19 patients.

\section{The expression of ACE2 in taste cells may be the potential mechanism of amblygeustia}

Another important finding of Chen et al. was that 14 types of oral symptoms were found in the COVID-19 patients, including amblygeustia, dry mouth, dryness and inflammation of mouth ${ }^{5}$. Among these oral symptoms, the amblygeustia was present in nearly half of the patients, with the percentage high to $47.2 \%{ }^{5}$. That the amblygeustia widely existed in the COVID-19 patients, were also demonstrated in the statement from the ENT UK and British Rhinological Society $(\mathrm{BRS})^{7}$. The oral symptoms of patients provided a new sight for screening the COVID-19 patients, especially for the patients without the common pneumonia symptoms.

To explore the potential mechanism of the amblygeustia in COVID-19 patients, we have performed further analysis with the single-cell profiles of tongue tissues. We chose the typical gene markers of taste buds to mark the taste cells in the tongue, which include TAS1R3, TAS2R4, TAS2R14, SNAP25 and NCAM1, and the cells with expression of any above genes would be generally regarded as taste cells ${ }^{8}$. The cellular distribution of taste cells and ACE2 positive cells was showed in Fig. 1, we found that the percentage of ACE2 positive cells in dorsum epithelial cells was $1.78 \%$, while the percentage in taste cells rose to $4.37 \%$, which showed that the ACE2 was inclined to express in taste cells. Details about the sample collection and data analysis could be found in our previous study ${ }^{3}$. This result indicated that the 2019nCoV may invade into taste cells at the early stage of COVID-19, and lead to the amblygeustia of patients.

\section{Conclusion}

Recent researches on the 2019-nCoV and COVID-19 in oral cavity are valuable and enlightening for future epidemic prevention strategy. The ACE2-expressing cells in oral epithelium and salivary glands, might provide possible routes of entry for the 2019-nCov. And the results that the 2019-nCoV could be detected in saliva not only validate the infectiousness of patients' saliva, also provide novel way to monitor the illness state and virus load of patients. The finding about amblygeustia of COVID-19 patients and its potential mechanism, indicate 
that besides the common clinical manifestations, such as fever and cough, we should also pay great attention to the oral symptoms of patients in dental settings, especially the amblygeustia. These findings suggest that oral examination and interrogation could be meaningful for preventing nosocomial infection and screening the asymptomatic patients of COVID-19 during the current epidemic.

\section{ACKNOWLEDGEMENTS}

This study was supported by grants from the National Natural Science Foundation of China (81520108009, 81991502, 81771081,81991500), the 111 Project of MOE (B14038), China.

\section{ADDITIONAL INFORMATION}

Competing interests: The authors declare no competing interests.

\section{References}

1 Epidemiology Working Group for NCIP Epidemic Response. The epidemiological characteristics of an outbreak of 2019 novel coronavirus diseases (COVID-19) in China. Chinese Journal of Epidemiology. 41, 7 (2020).

2 Zou, X. et al. Single-cell RNA-seq data analysis on the receptor ACE2 expression reveals the potential risk of different human organs vulnerable to 2019-nCoV infection. Front Med. (2020).

$3 \mathrm{Xu}, \mathrm{H}$. et al. High expression of ACE2 receptor of 2019-nCoV on the epithelial cells of oral mucosa. Int J Oral Sci. 12, 8 (2020).

4 To, K. K.-W. et al. Temporal profiles of viral load in posterior oropharyngeal saliva samples and serum antibody responses during infection by SARS-CoV-2: an observational cohort study. The Lancet Infectious Diseases. (2020).

5 Chen, L. et al. Detection of 2019-nCoV in Saliva and Characterization of Oral Symptoms in COVID-19 Patients. Available at SSRN 3557140. (2020).

6 To, K. K. et al. Consistent detection of 2019 novel coronavirus in saliva. Clin Infect Dis. (2020).

7 Hopkins, C. \& Kumar, N. Statement from ENT UK and BRS, $<$ https://www.fifthsense.org.uk/covid-19-corona-virus-and-smell-loss-guidance-from-fifthsense-medical-director/> (2020).

8 Roper, S. D. \& Chaudhari, N. Taste buds: cells, signals and synapses. Nature Reviews Neuroscience. 18, 485-497 (2017). 

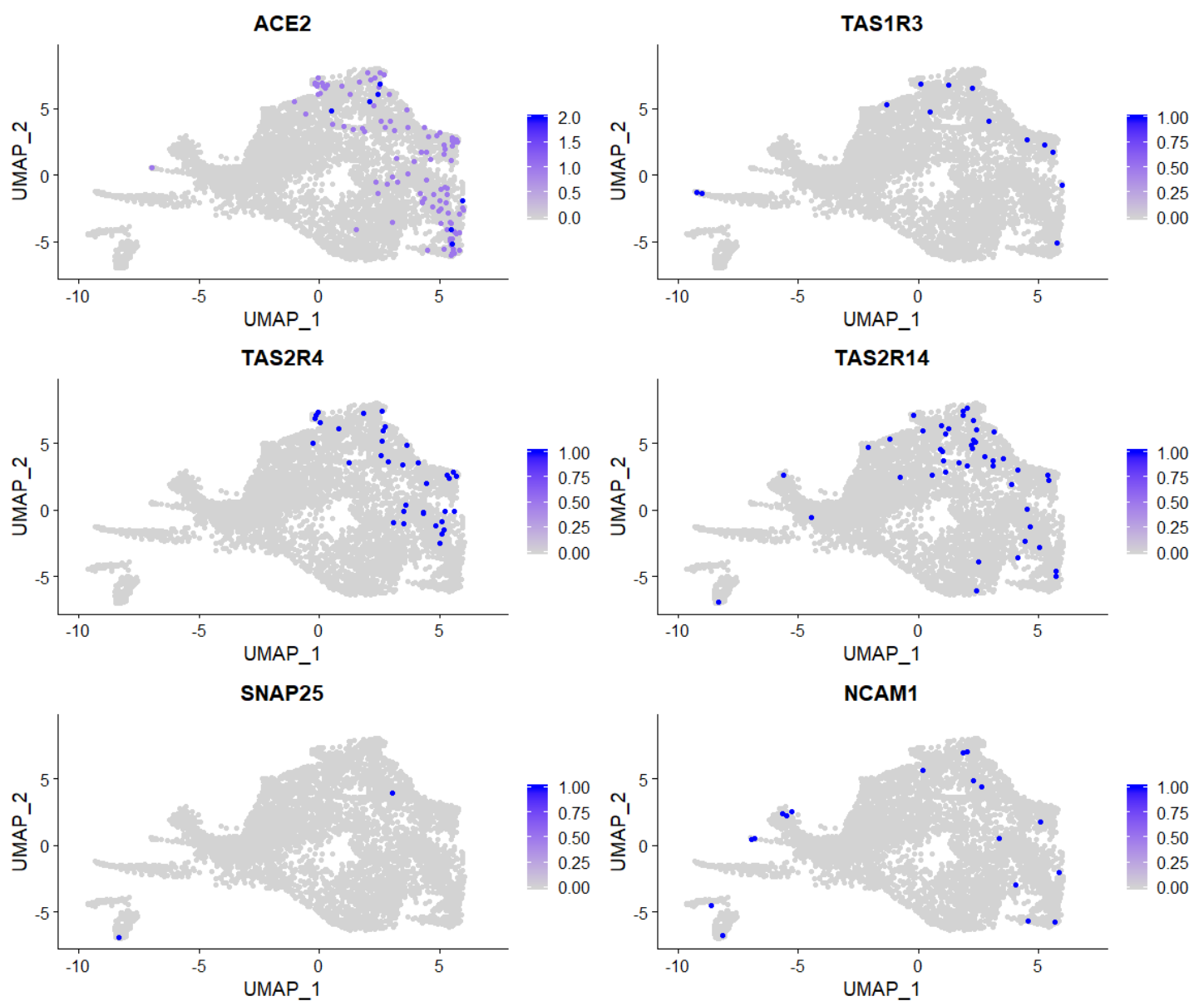

Figure 1. The distribution of ACE2 positive cells and taste-related gene marked cells 